\title{
Influence of Divalent Cations on Deformation and Rupture of Adsorbed Lipid Vesicles
}

\section{Supporting Information}

Marija Dacic ${ }^{1,2,+}$, Joshua A. Jackman ${ }^{1,2,+}$, Saziye Yorulmaz ${ }^{1,2}$, Vladimir P. Zhdanov ${ }^{1,2,4}$, Bengt Kasemo ${ }^{5}$, Nam-Joon Cho ${ }^{*, 1,2,3}$

${ }^{1}$ School of Materials Science and Engineering, Nanyang Technological University, 50 Nanyang Avenue 639798, Singapore

${ }^{2}$ Centre for Biomimetic Sensor Science, Nanyang Technological University, 50 Nanyang Drive 637553, Singapore

${ }^{3}$ School of Chemical and Biomedical Engineering, Nanyang Technological University, 62 Nanyang Drive 637459, Singapore

${ }^{4}$ Boreskov Institute of Catalysis, Russian Academy of Sciences, Novosibirsk 630090, Russia

${ }^{5}$ Department of Physics, Chalmers University of Technology, 41296 Göteborg, Sweden

${ }^{+}$The authors contributed equally to this work.

*Corresponding author

E-mail: njcho@ntu.edu.sg 


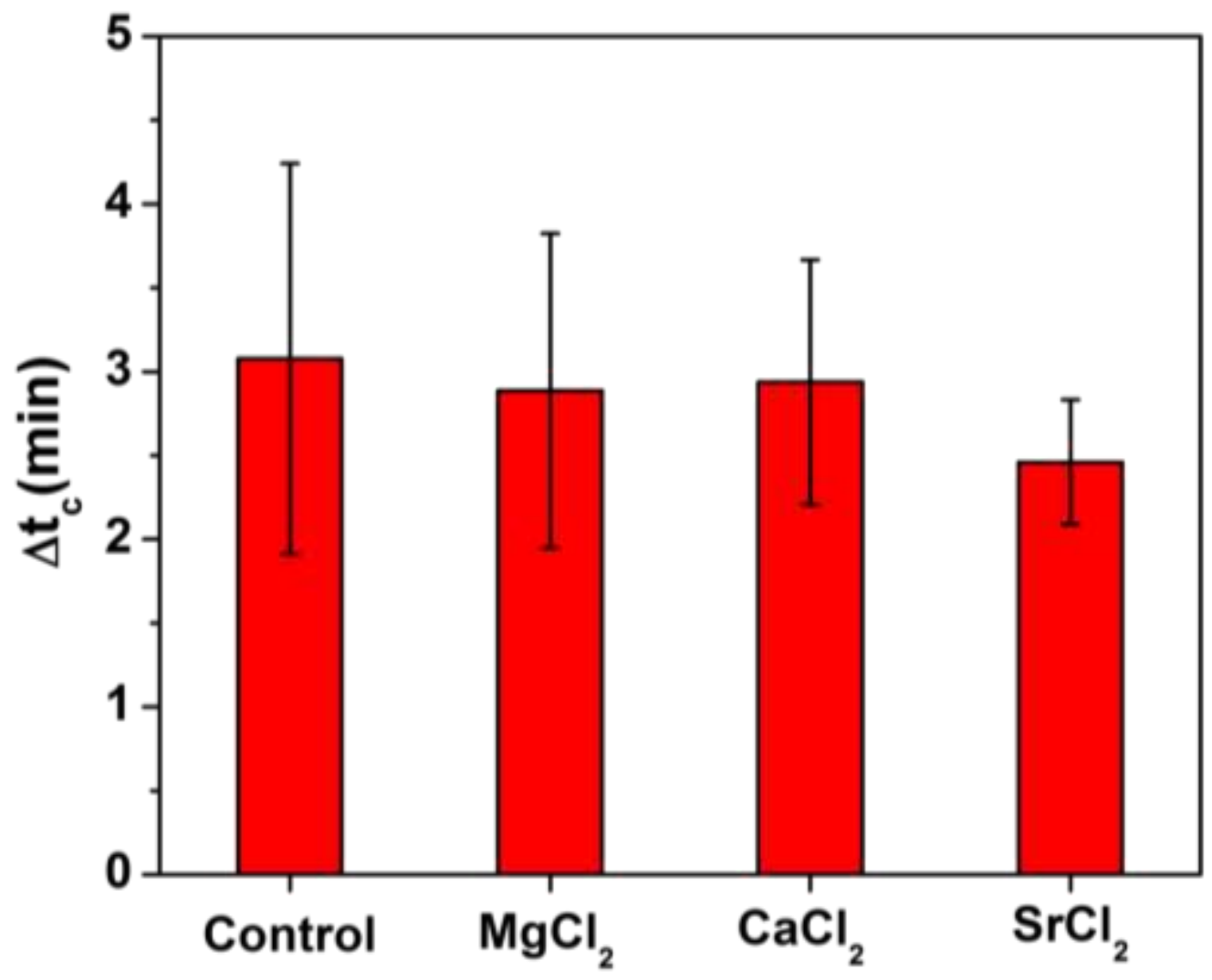

Figure S1. Effect of Divalent Cations on Kinetics of Vesicle Adsorption and Rupture on Silicon Oxide. QCM-D measurements were performed and $\Delta t_{c}$ values are presented as a function of time ( $\mathrm{n}=4$ measurements per condition). The control experiment contained the same vesicle preparation and buffer conditions without divalent cations. 


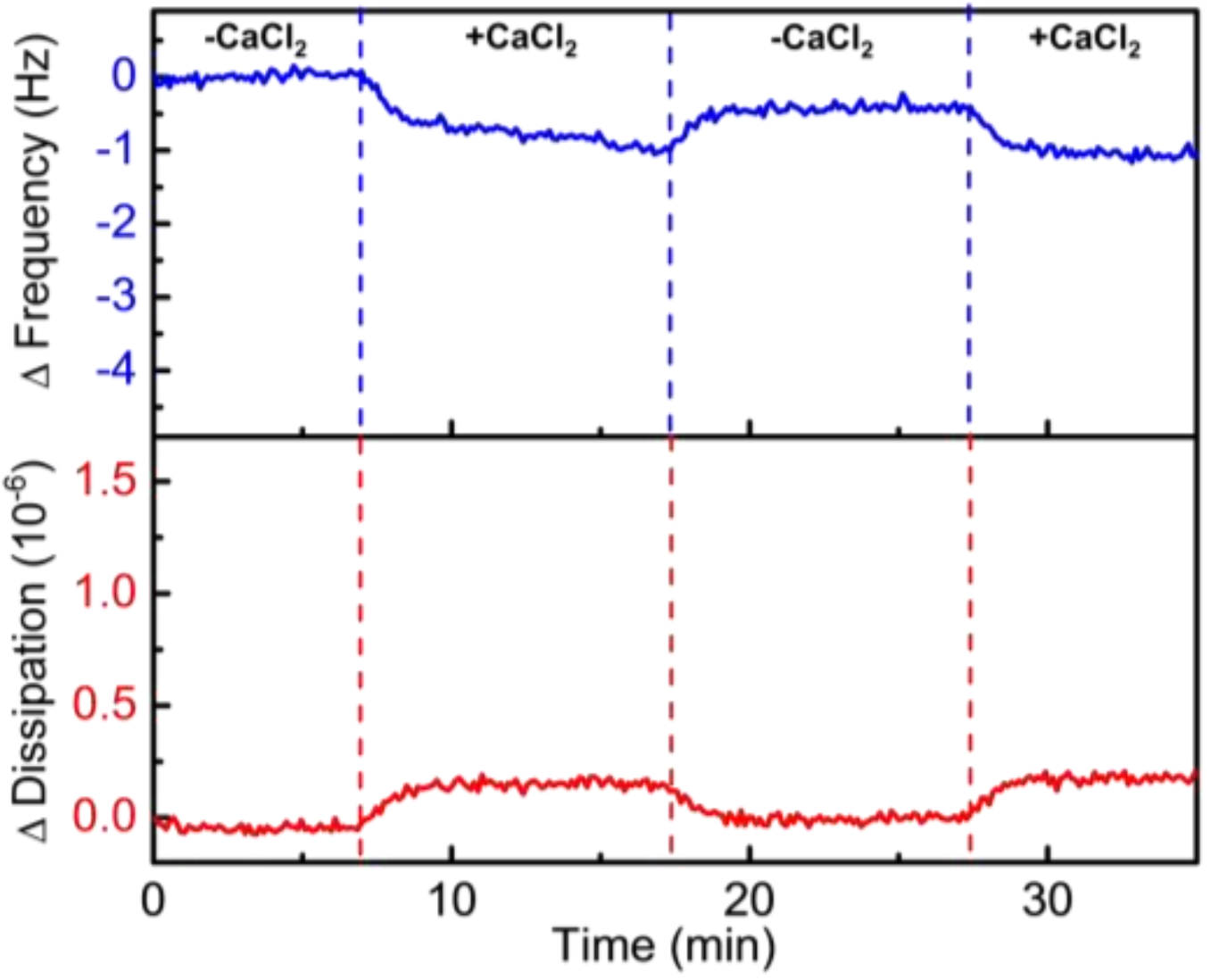

Figure S2. QCM-D Measurement Stability in the Presence and Absence of $5 \mathrm{mM} \mathrm{CaCl}_{2}$ on Silicon Oxide. QCM-D measurements were performed and the baseline signal was recorded in $10 \mathrm{mM}$ Tris buffer [pH 7.5] with $150 \mathrm{mM} \mathrm{NaCl}$ and no $\mathrm{CaCl}_{2}$, and then the solution buffer was exchanged back and forth approximately with the equivalent buffer that had $5 \mathrm{mM} \mathrm{CaCl}_{2}$. 


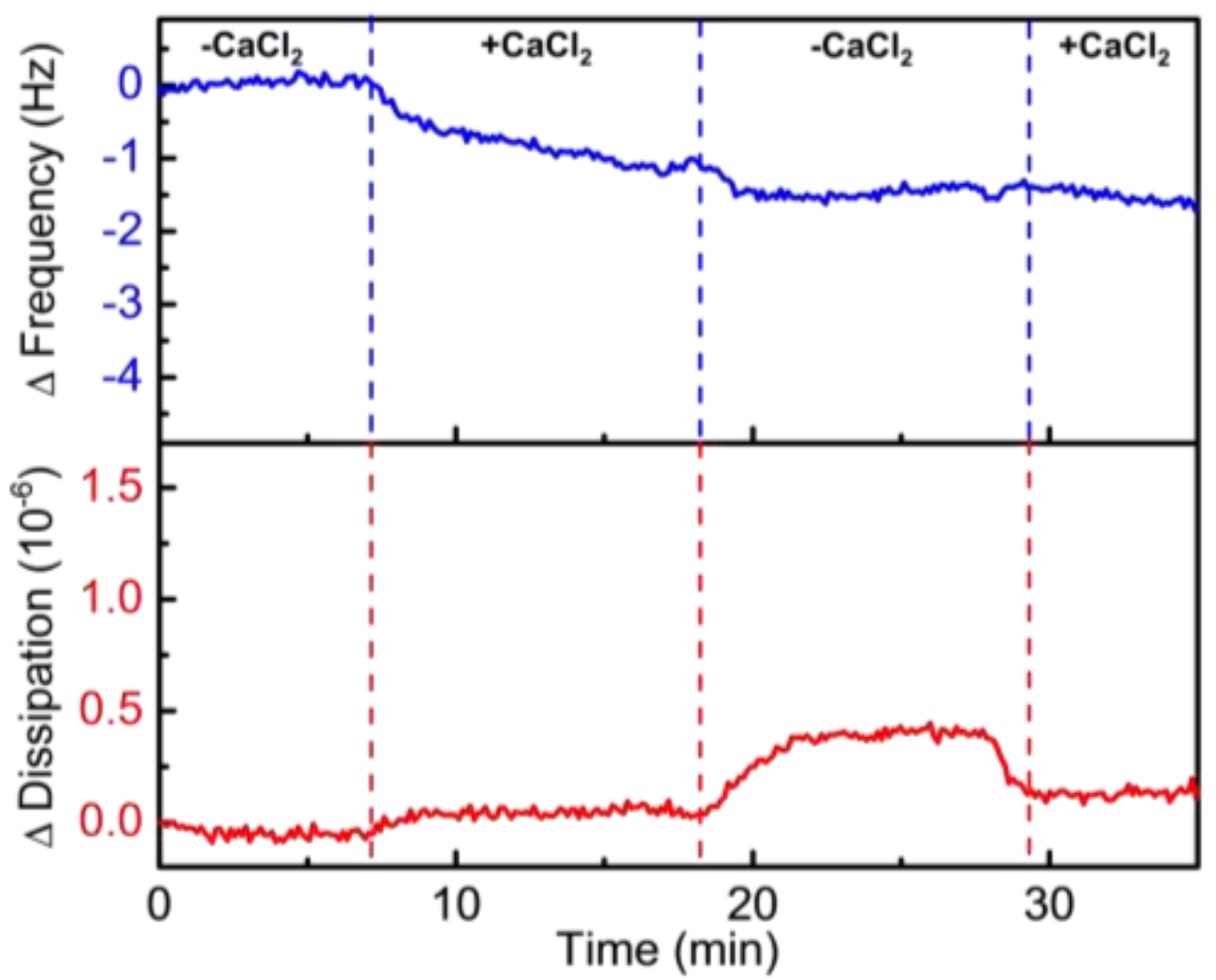

Figure S3. QCM-D Measurement Stability in the Presence and Absence of $5 \mathbf{m M ~} \mathbf{C a C l}_{2}$ on Titanium Oxide. QCM-D measurements were performed and the baseline signal was recorded in $10 \mathrm{mM}$ Tris buffer [pH 7.5] with $150 \mathrm{mM} \mathrm{NaCl}$ and no $\mathrm{CaCl}_{2}$, and then the solution buffer was exchanged back and forth approximately with the equivalent buffer that had $5 \mathrm{mM} \mathrm{CaCl}{ }_{2}$. 